\title{
Poster Abstract: Toward Multi-Task Support and Security Analyses in PLC Program Translation for Verification
}

\author{
Luis Garcia \\ Electrical and Computer Engineering \\ Department \\ University of California, Los Angeles \\ Los Angeles, CA, USA \\ garcialuis@ucla.edu
}

\author{
Stefan Mitsch \\ Computer Science Department \\ Carnegie Mellon University \\ Pittsburgh, PA, USA \\ smitsch@cs.cmu.edu
}

\author{
André Platzer \\ Computer Science Department \\ Carnegie Mellon University \\ Pittsburgh, PA, USA \\ aplatzer@cs.cmu.edu
}

\begin{abstract}
In this poster, we will present new tool, HyPLC [30], which implements the translation of discrete control code of verified hybrid program models to PLC controller code and, vice versa, the translation of existing PLC code into the discrete control actions for a hybrid program given an additional input of the continuous dynamics of the system to be verified. This approach allows for the generation of real controller code while preserving, by compilation, the correctness of a valid and verified hybrid program. PLCs are common cyber-physical interfaces for safety-critical industrial control applications, and HyPLC serves as a pragmatic tool for bridging formal verification of complex cyber-physical systems at the algorithmic level of hybrid programs with the execution layer of concrete PLC implementations. This poster will also discuss future directions of HyPLC such as support of multiple tasks (and, by extension, multiple PLCs), interval arithmetic, as well as security analyses.
\end{abstract}

\section{CCS CONCEPTS}

- Theory of computation $\rightarrow$ Formal languages and automata theory; Computing methodologies $\rightarrow$ Model verification and validation; $\bullet$ Computer systems organization $\rightarrow$ Embedded and cyber-physical systems; $\bullet$ Software and its engineering $\rightarrow$ Compilers.

\section{KEYWORDS}

Industrial control, programming languages, formal verification, semantics, compilation

\section{ACM Reference Format:}

Luis Garcia, Stefan Mitsch, and André Platzer. 2019. Poster Abstract: Toward Multi-Task Support and Security Analyses in PLC Program Translation for Verification. In 10th ACM/IEEE International Conference on Cyber-Physical Systems (with CPS-IoT Week 2019) (ICCPS '19), April 16-18, 2019, Montreal, QC, Canada. ACM, New York, NY, USA, 3 pages. https://doi.org/10.1145/ 3302509.3313335

Permission to make digital or hard copies of part or all of this work for personal or classroom use is granted without fee provided that copies are not made or distributed for profit or commercial advantage and that copies bear this notice and the full citation on the first page. Copyrights for third-party components of this work must be honored

For all other uses, contact the owner/author(s).

ICCPS '19, April 16-18, 2019, Montreal, QC, Canada

(c) 2019 Copyright held by the owner/author(s).

ACM ISBN 978-1-4503-6285-6/19/04.

https://doi.org/10.1145/3302509.3313335

\section{HYPLC OVERVIEW}

There has been an increased emphasis on the verification and validation of software used in embedded systems in the context of industrial control systems (ICS). ICS represent a class of cyber-physical systems (CPS) that provide monitoring and networked process control for safety-critical industrial environments, e.g., the electric power grid [1], railway safety [2], nuclear reactors [3], and water treatment plants [4]. A prominent choice of implementation platform for many ICS applications are programmable logic controllers (PLCs) that act as interfaces between the cyber world-i.e., the monitoring entities and process control-and the physical world-i.e., the underlying physical system that the ICS is sensing and actuating. Efforts to verify the correctness of PLC applications focus on the code that is loaded onto these controllers [5-8].. Existing methods are based on model checking of safety properties specified in modal temporal logics, e.g., Linear Temporal Logic (LTL) [9] and Computation Tree Logic (CTL) [10]. However, since PLC code does not include a model of the system plant, such analyses are limited to more superficial, discrete properties of the code instead of analyzing safety properties of the resulting physical behavior.

In this poster, we will present our approach that starts from hybrid systems models of ICS, in which the discrete computations of controllers run together with the continuous evolution of the underlying physical system. That way, correctness properties that consider both control decisions and physical evolution can be verified in the theorem prover KeYmaera X [11]. The verified hybrid programs can then be compiled to PLC code and executed as controllers. The reverse compilation from PLC code to hybrid programs facilitates verifying existing PLC code with respect to pre-defined models of the continuous plant dynamics.

Our tool, HyPLC, compiles verified hybrid systems models into PLC code and vice versa. Figure 1 depicts a high-level overview of the bidirectional compilation provided by HyPLC. The hybrid models are specified in differential dynamic logic, $\mathrm{dL}$ [12-14], which is a dynamic logic for hybrid systems expressed as hybrid programs. Compiling hybrid programs to PLC code generates deterministic implementations of the controller abstractions typically found in hybrid programs, which focus on capturing the safety-relevant decisions for verification purposes concisely with nondeterministic modeling concepts. Nondeterminism in hybrid programs can be beneficial for verification since nondeterministic models address a family of (control) programs with a single proof at once, but is detrimental to implementation with Structured Text (ST) programs on PLCs. Therefore, in this work we focus on hybrid programs in scan cycle form. The compilation adopts the IEC 61131-3 standards 


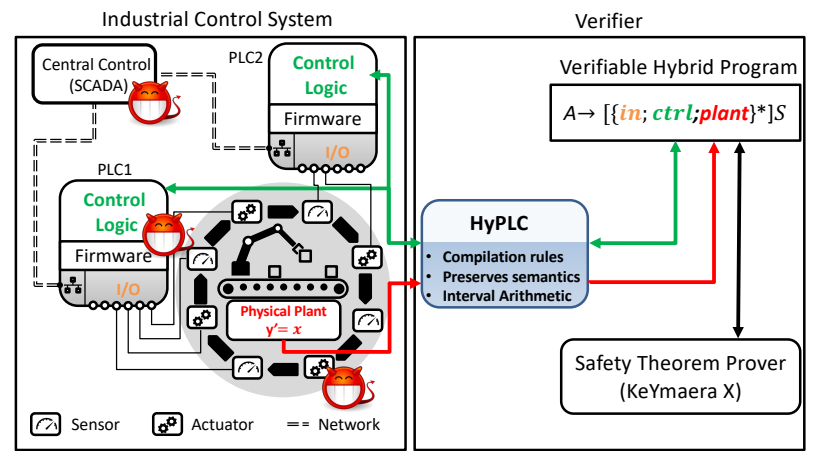

Figure 1: HyPLC provides a bidirectional translation of the discrete control of a verifiable hybrid program expressed in $\mathrm{dL}$ and the control logic code that runs on a PLC in the context of a cyber-physical industrial control system. Future work will focus on support of multiple tasks (and, by extension, multiple PLCs), interval arithmetic, as well as security analyses.

for PLCs [15]. Compiling PLC code to dL and hybrid programs, implemented using the ANTLR parser generator [16], provides a means of analyzing PLC code on pre-defined models of continuous evolution with the deductive verification techniques of KeYmaera X. The core contributions of HyPLC lie in our correctness proofs for the bidirectional compilation, so that both directions of compilation yield a way of obtaining code with safety guarantees (assuming no floating-point arithmetic errors arise).

\section{FUTURE WORK}

In this poster, we plan to demo the current state of HyPLC, which contains the contributions enumerated in the previous section. However, we also plan on discussing main directions of future research-lifting assumptions, support for multiple tasks, and support for security analysis.

Interval arithmetic. A main assumption of HyPLC is that the arithmetic operations phrased in hybrid programs over real arithmetic translate to the machine arithmetic available in PLC. Interval arithmetic, as used in the VeriPhy compilation pipeline [?], presents an approach to soundly implement arithmetic.

Multi-task support. Currently, HyPLC supports a single configuration for a single resource (PLC) running a single task. A future extension to multiple tasks may consider a single configuration of a PLC with a single resource that has a one-to-one mapping of task configurations to ST programs. A designated clock $t_{n}$ per task would keep track of the associated task's execution interval $\varepsilon_{n}$. The task execution intervals would be checked periodically every $\varepsilon_{\mathrm{sc}}$ times, which represents the scan cycle timing of the PLC. Any task with elapsed clock $t_{n} \geq \varepsilon_{n}$ is executed (which means that tasks are executed with at most $\varepsilon_{\mathrm{sc}}$ delay). This requires a correct proof of encoding for both multiple tasks as well as a priority structure.

Security analysis. Prior works have analyzed the cyber-physical effects of "mutating" lines of code on a PLC [?]. These code mutations are used to learn anomalous behavior of the PLC. However, the choice of the lines of code to mutate as well as the choice of PLC are completely random and arbitrary. HyPLC could be used to complement such an approach by observing how such a mutation would effect the safety of the system given a sound translation. Similarly, HyPLC could complement cyber-physical intrusion detection solutions that depend on state estimation [?].

\section{ACKNOWLEDGMENTS}

This research was sponsored by the U.S. Department of Education under the Graduate Assistance in Areas of National Need Fellowship, the U.S. Department of Energy under the grant number DEOE0000780, the Air Force Office of Scientific Research (AFOSR) under the grant number FA9550-16-1-0288, as well as the Defense Advanced Research Projects Agency (DARPA) Grant Number FA875018-C-0092. The views and conclusions contained in this document are those of the author and should not be interpreted as representing the official policies, either expressed or implied, of any sponsoring institution, the U.S. government or any other entity.

\section{REFERENCES}

[1] L. Garcia, S. Mitsch, and A. Platzer, "HyPLC: Hybrid programmable logic controller program translation for verification," CoRR, vol. abs/1902.05205, 2019.

[2] M. F. McGranaghan, D. R. Mueller, and M. J. Samotyj, "Voltage sags in industrial systems," IEEE Transactions on industry applications, vol. 29, no. 2, pp. 397-403, 1993.

[3] "ABB launches new Pluto programmable logic controller for rail safety applications." [Online]. Available: http://www.abb.com/cawp/seitp202/ fa405fb9803dd9eac1258035002f53c0.aspx

[4] B. Kesler, "The vulnerability of nuclear facilities to cyber attack; strategic insights: Spring 2010," Strategic Insights, Monterey, California. Naval Postgraduate School, Spring 2011, 2011.

[5] S. Manesis, D. Sapidis, and R. King, "Intelligent control of wastewater treatment plants," Artificial Intelligence in Engineering, vol. 12, no. 3, pp. 275-281, 1998.

[6] I. Moon, "Modeling programmable logic controllers for logic verification," IEEE Control Systems, vol. 14, no. 2, pp. 53-59, 1994.

[7] D. Darvas, E. Blanco Vinuela, and I. Majzik, "A formal specification method for PLC-based applications," in 15th International Conference on Accelerator and Large Experimental Physics Control Systems. JACoW, 2015, pp. 907-910.

[8] A. Mader and H. Wupper, "Timed automaton models for simple programmable logic controllers," in Real-Time Systems, 1999. Proceedings of the 11th Euromicro Conference on. IEEE, 1999, pp. 106-113.

[9] D. Thapa, S. Dangol, and G.-N. Wang, "Transformation from Petri nets model to programmable logic controller using one-to-one mapping technique," in Computational Intelligence for Modelling, Control and Automation, 2005 and International Conference on Intelligent Agents, Web Technologies and Internet Commerce, International Conference on, vol. 2. IEEE, 2005, pp. 228-233.

[10] R. Gerth, D. Peled, M. Y. Vardi, and P. Wolper, "Simple on-the-fly automatic verification of linear temporal logic," in Protocol Specification, Testing and Verification $X V$. Springer, 1995, pp. 3-18.

[11] E. M. Clarke, E. A. Emerson, and A. P. Sistla, "Automatic verification of finite-state concurrent systems using temporal logic specifications," ACM Transactions on Programming Languages and Systems (TOPLAS), vol. 8, no. 2, pp. 244-263, 1986.

[12] N. Fulton, S. Mitsch, J-D. Quesel, M. Völp, and A. Platzer, "KeYmaera X: an axiomatic tactical theorem prover for hybrid systems," in International Conference on Automated Deduction. Springer, 2015, pp. 527-538.

[13] A. Platzer, "Differential dynamic logic for hybrid systems." 7. Autom. Reas., vol. 41, no. 2 , pp. $143-189,2008$

[14] --, "A complete uniform substitution calculus for differential dynamic logic," 7 . Autom. Reas., vol. 59, no. 2, pp. 219-265, 2017.

[15] --, Logical Foundations of Cyber-Physical Systems. Switzerland: Springer, 2018.

[16] K.-H. John and M. Tiegelkamp, IEC 61131-3: programming industrial automation systems: concepts and programming languages, requirements for programming systems, decision-making aids. Springer Science \& Business Media, 2010.

[17] "Antlr." [Online]. Available: https://www.antlr.org/

[18] B. Bohrer, Y. K. Tan, S. Mitsch, M. O. Myreen, and A. Platzer, "VeriPhy: Verified controller executables from verified cyber-physical system models," in PLDI, D. Grossman, Ed. ACM, 2018, pp. 617-630.

[19] Y. Chen, C. M. Poskitt, and J. Sun, "Learning from mutants: Using code mutation to learn and monitor invariants of a cyber-physical system," in 2018 IEEE Symposium on Security and Privacy (SP), vol. 00, 2018, pp. 240-252. [Online]. Available: doi.ieeecomputersociety.org/10.1109/SP.2018.00016

[20] S. Adepu, F. Brasser, L. Garcia, M. Rodler, L. Davi, A.-R. Sadeghi, and S. Zonouz, "Control behavior integrity for distributed cyber-physical systems," arXiv preprint arXiv:1812.08310, 2018. 